\title{
Screening multivariado entre acessos e cultivares de feijão do grupo preto para utilização em blocos de cruzamento
}

\author{
Diego Stähelin ${ }^{1 *}$ \\ Giseli Valentini ${ }^{1}$ \\ Luciano Rogério Braatz de Andrade ${ }^{1}$ \\ Mario Alvaro Aloisio Verissimo ${ }^{1}$ \\ Juliano Garcia Bertoldo ${ }^{2}$ \\ Altamir Frederico Guidolin ${ }^{1}$ \\ Jefferson Luis Meirelles Coimbra ${ }^{1}$
${ }^{1}$ PPG em Produção Vegetal, Instituto de Melhoramento e Genética Molecular Departamento de Agronomia, Universidade do Estado de Santa Catarina Avenida Camões 2090, CEP 88520-000, Lages - SC, Brasil ${ }^{2} \mathrm{PPG}$ em Recursos Genéticos Vegetais, Centro de Ciências Agrárias Universidade Federal de Santa Catarina *Autor para correspondência diegostahelin@gmail.com

\section{Resumo}

A estatística multivariada, através de medidas de dissimilaridade, vem auxiliando melhoristas de plantas na identificação de genótipos contrastantes utilizados na obtenção de populações segregantes de maior variabilidade. O objetivo do presente trabalho foi avaliar a dissimilaridade entre 38 acessos de feijão do grupo preto, por meio de técnicas multivariadas e selecionar genitores para compor blocos de cruzamentos. A partir das distâncias Euclidianas entre os acessos, realizou-se o agrupamento pelo método hierárquico de Ward, o qual permitiu classificar os acessos em grupos divergentes, sendo posteriormente comparados através de contrastes multivariados. Com a utilização dos escores das três primeiras variáveis canônicas foi possível a formação de seis grupos distintos que corroboram e se assemelham à análise por métodos aglomerativos. Os resultados revelaram variabilidade entre os acessos estudados, onde a análise multivariada pode adicionar informações à variação conjunta de variáveis respostas, sendo eficiente na determinação de divergência entre tais acessos.

Palavras-chave: Phaseolus vulgaris L., Técnicas de agrupamento, Variáveis canônicas

\section{Abstract}

Multivariate screening among black beans accessions to participation in crossing blocks. The multivariate statistical analysis, through measures of dissimilarity, has been helping plant breeders in identifying distant genotypes used to obtain populations segregating desired. Thus, the objective of this study was to evaluate the dissimilarity between 38 accessions of black beans by means of multivariate techniques, and to select parents 
to compose blocks of crosses. From the Euclidean distance between accessions, grouping by the hierarchical method of Ward was carried out to permit classification of the accessions into different groups, which were then compared using multivariate contrasts. With the use of scores of the first three canonical variables, it was then possible to form six distinct groups that were similar and to corroborate the analysis by clustering methods. The results revealed a wide variability among the accessions studied, where the multivariate analysis was able to add information to the joint range of response variables, and was efficient in determining the difference between such accessions.

Key words: Canonical variables, Phaseolus vulgaris L., Techniques of grouping

\section{Introdução}

No planejamento de um programa de melhoramento de plantas é comum considerar a cultivar a ser produzida como uma substituição de alguma variedade que já vem sendo cultivada. Desta forma, quase sem exceção, um dos pais é escolhido em função do seu desempenho comprovado nas áreas onde se pretende usar a nova variedade. O outro genitor é escolhido por complementar deficiências específicas do primeiro genitor (ALLARD, 1999).

Assim, uma das etapas iniciais e fundamentais para o sucesso de um programa de melhoramento consiste na escolha dos genitores que formarão a população segregante (CARVALHO et al., 2003; BORÉM; MIRANDA, 2009). Um dos procedimentos estatísticos para a estimativa dos melhores genitores para cruzamentos em programas de melhoramento a disposição dos pesquisadores é a estatística multivariada.

A estatística multivariada é definida como um conjunto de métodos estatísticos utilizados em situações nas quais varias variáveis, contínuas ou não, são medidas simultaneamente em cada unidade experimental. Os métodos de análise de dados multivariados permitem um estudo global dessas variáveis, colocando em evidência as ligações, semelhanças ou diferenças entre elas, com perdas mínimas de informação (SARTORIO, 2008). De acordo com o mesmo autor, a principal justificativa para a utilização da análise multivariada é o fato de nenhuma variável conseguir, individualmente, caracterizar de maneira adequada a unidade experimental ou mesmo discriminar os fatores em estudo com relação a qualquer critério empregado individualmente na análise. Sendo assim, em experimentos que envolvem duas ou mais variáveis dependentes, o procedimento adequado para a inferência estatística é a análise multivariada.

Algumas técnicas multivariadas podem ser aplicadas na predição da divergência em populações, como variáveis canônicas e métodos aglomerativos, classificadas como técnicas exploratórias por promoverem a simplificação da estrutura de variabilidade dos dados (MINGOTI, 2005). Carvalho et al. (2003) comentam que os métodos preditivos de dissimilaridade têm sido bastante utilizados no melhoramento vegetal, sobretudo pelo fato de que, ao se basearem em diferenças morfológicas e fisiológicas dos genitores, dispensam a obtenção das combinações híbridas entre eles, o que é vantajoso, especialmente quando o número de genitores cujas divergências se deseja conhecer, é elevado.

Desta forma, a análise da divergência vem sendo utilizada por melhoristas de plantas para investigar relações de parentesco, diversidade de origem geográfica, capacidade de combinação e heterose nas espécies em estudo (CRUZ, 1990). Neste sentido as avaliações de divergência objetivam fornecer parâmetros para identificação de genitores que propiciam maior variabilidade nas gerações segregantes quando combinados em hibridações e consequentemente maior possibilidade de recuperação de genótipos superiores (CRUZ et al., 1994a; 1994b; FALCONER; MACKAY, 1996; CRUZ; REGAZZI, 1997; DIAS; KAGEYAMA, 1997). Tal fenômeno, conhecido como segregação transgressiva, vem sendo relado em espécies autógamas para características como resistência quantitativa a doenças (WESP et al., 2008; SILVA et al., 2008), qualidade de grãos (KUREK et al., 2002; RONALD et al., 1999), ciclo cultural (FIORINI et al. 2005; CARVALHO FILHO et al., 2009) e produtividade de grãos (MENDONÇA et al., 2002; MATEI, 2010). 
Assim, o presente trabalho teve como objetivo avaliar a dissimilaridade entre acessos de feijão do Banco Ativo de Germoplasma de Feijão da Universidade do Estado de Santa Catarina, com a utilização da estatística multivariada, visando à incorporação de genitores com características desejáveis em blocos de cruzamento dirigido.

\section{Material e Métodos}

O experimento foi conduzido no ano agrícola de 2008/2009 na área experimental do Instituto de Melhoramento e Genética Molecular da Universidade do Estado de Santa Catarina (IMEGEM/UDESC), em Lages, situada a $27^{\circ} 47^{\prime} \mathrm{S}$ de latitude, $50^{\circ} 18^{\prime} \mathrm{W}$ de longitude e $916 \mathrm{~m}$ de altitude.

Foram semeados 38 acessos crioulos (landraces) de feijão do grupo comercial preto, doados por agricultores ao Banco Ativo de Germoplasma de Feijão da UDESC (Tabela 1). A densidade de semeadura foi de 12 sementes por metro linear. A parcela foi constituída de 4 fileiras, com $4 \mathrm{~m}$ de comprimento e espaçamento entre fileiras de $0,45 \mathrm{~m}$. A área útil foi composta por duas fileiras centrais, descartando-se $0,5 \mathrm{~m}$ das extremidades. $\mathrm{O}$ experimento foi conduzido sob condições naturais de precipitação pluvial. $\mathrm{O}$ delineamento utilizado foi o de blocos aumentados (FEDERER, 1956) com 2 testemunhas comerciais intercaladas, com 11 repetições, para estimativa do resíduo experimental (BRS Supremo, IPR Uirapuru).

O solo da área experimental é classificado como CAMBISSOLO HÚMICO distrófico(EMBRAPA,1999), apresentando as seguintes características: $40 \%$ de argila; pH em água 5,6 e pH SMP 6,2; 3,2mg dm ${ }^{-3} \mathrm{de}$ P; $62 \mathrm{mg}$ $\mathrm{dm}^{-3}$ de $\mathrm{K} ; 3,9 \%$ de MO; $5 \mathrm{cmol}_{\mathrm{c}} \mathrm{dm}^{-3}$ de Ca; e 2,8 $\mathrm{cmol}_{\mathrm{c}}$ $\mathrm{dm}^{-3} \mathrm{de} \mathrm{Mg}$. A adubação foi realizada de acordo com as recomendações para a cultura (COMISSÃO SULBRASILEIRA DE QUÍMICA E FERTILIDADE DO SOLO, 2004) e constou da aplicação de $20 \mathrm{~kg} \mathrm{ha}^{-1}$ de $\mathrm{N}, 80 \mathrm{~kg} \mathrm{ha}^{-1}$ de $\mathrm{P}_{2} \mathrm{O}_{5}$ e $70 \mathrm{~kg} \mathrm{ha}^{-1}$ de $_{2} \mathrm{O}$, aplicados na base, nas formas de uréia, superfosfato triplo e cloreto de potássio. O Nitrogênio em cobertura foi aplicado na forma de uréia, nas doses de $35 \mathrm{~kg} \mathrm{ha}^{-1}$ e $30 \mathrm{~kg} \mathrm{ha}^{-1}$ aos 20 dias após a emergência e no estádio R5 (início do florescimento), respectivamente. O controle de plantas invasoras foi realizado através de capina manual e o controle de insetos pela aplicação de $500 \mathrm{~g} \mathrm{ha}^{-1}$ de Metamidophos (Tamaron BR).

Durante o desenvolvimento da cultura e após sua colheita foram realizadas avaliações morfoagronomicas seguindo-se descritores específicos da cultura, tomando-se aleatóriamente 10 plantas dentro de cada parcela (CIAT, 1991; IPGRI, 2001; SILVA, 2005). Nas análises realizadas foram utilizados onze caracteres: produtividade de grãos em $\mathrm{kg} \mathrm{ha}^{-1}$ (PROD), massa de cem grãos (MCG), número de dias entre a emergência das plântulas e a floração (DEF), número de dias entre a floração e a maturação fisiológica (DFM), estatura de plantas (EP), altura de inserção do primeiro legume (IPL), número de legumes por planta (NLP), número de grãos por legume (NGL), diâmetro da região do colo do caule (DRC), comprimento do folíolo principal da terceira folha trifoliolada (CFT) e largura do folíolo principal da terceira folha trifoliolada (LFT).

Os dados foram submetidos à análise de variância univariada pelo procedimento de modelos lineares generalizados (SAS INSTITUTE INC., 1985) para estimativa das médias ajustadas e posterior análise multivariada pelo procedimento PROC CANDISC (KHATTREE; NAIK, 1999) através de variáveis canônicas e agrupamento realizado pelo método de Ward (1963), utilizando como medida da dissimilaridade a distância Euclidiana. A padronização das variáveis estudadas é realizada pelo procedimento CANDISC, não havendo a necessidade de uma padronização $a$ priori (KHATTREE; NAIK, 1999). Com base no grau de similaridade a partir da referida matriz de distância entre os acessos foi construído um dendrograma com a utilização do procedimento PROC CLUSTER (STATISTICAL ANALYSIS SYSTEM, 2002). 
TABELA 1: Médias ajustadas de onze características avaliadas de trinta e oito acessos e duas cultivares comerciais de feijão de grão tipo preto (Lages-SC, 2008/2009).

\begin{tabular}{|c|c|c|c|c|c|c|c|c|c|c|c|c|c|}
\hline \multirow{2}{*}{$\begin{array}{l}\text { ACESSO/ } \\
\text { GENÓTIPO }\end{array}$} & \multicolumn{2}{|c|}{ DADOS DE PASSAPORTE } & \multicolumn{11}{|c|}{ MÉDIAS DOS CARACTERES AVALIADOS ${ }^{1 /}$} \\
\hline & ORIGEM & CATEGORIA $^{3 /}$ & PROD & MCG & DEF & DFM & EP & IPL & NLP & NGL & DRC & CFT & LFT \\
\hline BAF01 & Ponte Serrada/SC & $\mathrm{CT}$ & 3697,0 & 23,6 & 36,0 & 46,0 & 71,5 & 23,9 & 16,7 & 3,8 & 0,50 & 9,9 & 7,7 \\
\hline BAF02 & Pelotas/RS & $\mathrm{CT}$ & 4145,0 & 24,2 & 34,0 & 47,0 & 81,1 & 15,5 & 15,0 & 4,3 & 0,47 & 10,0 & 7,2 \\
\hline BAF07 & Lages/SC & $\mathrm{CT}$ & 2597,0 & 17,2 & 47,0 & 49,0 & 98,1 & 28,7 & 19,4 & 4,0 & 0,58 & 10,2 & 8,1 \\
\hline BAF08 & Erechim/RS & $\mathrm{CT}$ & 2148,0 & 43,1 & 33,0 & 36,0 & 62,1 & 20,8 & 7,6 & 3,3 & 0,38 & 10,4 & 7,8 \\
\hline BAF09 & Piracicaba/SP & $\mathrm{CT}$ & 4042,0 & 26,6 & 41,0 & 50,0 & 105,8 & 25,9 & 14,9 & 5,4 & 0,42 & 10,3 & 8,2 \\
\hline BAF23 & Chapecó/SC & $\mathrm{CT}$ & 2583,0 & 23,4 & 38,0 & 44,0 & 65,0 & 23,3 & 9,0 & 5,7 & 0,55 & 8,2 & 6,1 \\
\hline BAF25 & Palmitos/SC & $\mathrm{CT}$ & 3188,0 & 20,5 & 38,0 & 44,0 & 70,8 & 24,7 & 16,3 & 3,8 & 0,53 & 12,6 & 8,6 \\
\hline BAF26 & Palmitos/SC & $\mathrm{CT}$ & 3186,0 & 22,5 & 37,0 & 47,0 & 69,7 & 19,8 & 10,4 & 4,2 & 0,52 & 11,3 & 8,5 \\
\hline BAF36 & S. José do Cerrito/SC & $\mathrm{CT}$ & 2518,0 & 33,9 & 30,0 & 42,0 & 74,1 & 16,8 & 7,7 & 3,9 & 0,43 & 11,7 & 8,9 \\
\hline BAF38 & Pelotas/RS & $\mathrm{CT}$ & 2506,0 & 23,7 & 36,0 & 49,0 & 69,9 & 19,2 & 13,8 & 4,8 & 0,51 & 12,2 & 9,4 \\
\hline BAF40 & Capão Alto/SC & $\mathrm{CT}$ & 3458,0 & 23,8 & 35,0 & 48,0 & 71,2 & 16,5 & 11,5 & 4,6 & 0,43 & 10,9 & 10,0 \\
\hline BAF46 & Lages/SC & $\mathrm{CT}$ & 2902,0 & 22,5 & 41,0 & 49,0 & 96,9 & 30,5 & 9,2 & 4,8 & 0,47 & 10,4 & 7,6 \\
\hline BAF47 & Piratuba/SC & $\mathrm{CT}$ & 3247,0 & 22,4 & 37,0 & 46,0 & 84,9 & 25,7 & 11,2 & 5,9 & 0,48 & 10,2 & 7,3 \\
\hline BAF55 & Cunha Porã/SC & $\mathrm{CT}$ & 1332,0 & 18,9 & 36,0 & 45,0 & 75,7 & 23,5 & 14,4 & 4,8 & 0,55 & 8,0 & 7,5 \\
\hline BAF56 & Cunha Porã/SC & $\mathrm{CT}$ & 1760,0 & 21,6 & 31,0 & 55,0 & 64,1 & 20,3 & 12,5 & 4,2 & 0,45 & 10,5 & 7,7 \\
\hline BAF57 & Cunha Porã/SC & $\mathrm{CT}$ & 2127,0 & 21,5 & 35,0 & 53,0 & 75,7 & 20,1 & 21,3 & 4,8 & 0,65 & 9,3 & 7,7 \\
\hline BAF60 & Lebon Regis/SC & $\mathrm{CT}$ & 2645,0 & 20,0 & 37,0 & 48,0 & 75,0 & 18,7 & 13,4 & 5,6 & 0,48 & 10,0 & 6,9 \\
\hline BAF74 & Irineópolis/SC & CT & 3429,0 & 18,2 & 38,0 & 47,0 & 69,2 & 27,8 & 11,4 & 3,5 & 0,46 & 9,9 & 6,9 \\
\hline BAF75 & Formigueiro/RS & $\mathrm{CT}$ & 2440,0 & 20,3 & 34,0 & 48,0 & 57,8 & 16,4 & 11,8 & 4,5 & 0,43 & 11,4 & 8,2 \\
\hline BAF80 & Fraiburgo/SC & $\mathrm{CT}$ & 1839,0 & 37,5 & 28,0 & 51,0 & 104,9 & 28,0 & 14,0 & 5,2 & 0,45 & 8,7 & 7,5 \\
\hline BAF81 & Lebon Regis/SC & $\mathrm{CT}$ & 2281,0 & 19,5 & 43,0 & 42,0 & 92,6 & 33,7 & 12,3 & 4,4 & 0,46 & 8,2 & 6,1 \\
\hline BAF88 & Curitibanos/SC & $\mathrm{CT}$ & 3455,0 & 25,2 & 37,0 & 47,0 & 66,7 & 19,5 & 16,7 & 5,2 & 0,46 & 10,1 & 8,0 \\
\hline BAF97 & Iraí/RS & $\mathrm{CT}$ & 2257,0 & 24,8 & 33,0 & 48,0 & 73,9 & 21,1 & 8,7 & 3,8 & 0,39 & 8,9 & 6,8 \\
\hline BAF98 & Bagé/RS & $\mathrm{CT}$ & 3126,0 & 20,4 & 34,0 & 43,0 & 56,9 & 15,4 & 13,6 & 5,0 & 0,48 & 8,5 & 7,6 \\
\hline BAF 102 & Goiania/GO & CT & 2533,0 & 23,0 & 35,0 & 45,0 & 84,8 & 14,7 & 12,0 & 4,9 & 0,43 & 11,3 & 8,2 \\
\hline BAF114 & Capão Alto/SC & $\mathrm{CT}$ & 3152,0 & 34,3 & 32,0 & 40,0 & 58,6 & 16,0 & 6,9 & 4,0 & 0,42 & 8,5 & 6,7 \\
\hline BAF127 & Riqueza/SC & $\mathrm{CT}$ & 3395,0 & 37,8 & 30,0 & 42,0 & 61,8 & 17,5 & 9,3 & 3,5 & 0,38 & 7,2 & 5,1 \\
\hline BAF130 & Anchieta/SC & $\mathrm{CT}$ & 1795,0 & 16,7 & 35,0 & 46,0 & 69,0 & 20,2 & 15,1 & 4,1 & 0,58 & 10,4 & 7,5 \\
\hline BAF131 & Anchieta/SC & $\mathrm{CT}$ & 2380,0 & 20,8 & 39,0 & 46,0 & 80,6 & 38,1 & 9,5 & 4,9 & 0,48 & 8,3 & 6,6 \\
\hline BAF140 & Lacerdópolis/SC & $\mathrm{CT}$ & 2711,0 & 39,1 & 30,0 & 40,0 & 52,9 & 14,6 & 6,7 & 3,1 & 0,35 & 9,9 & 7,7 \\
\hline BAF150 & Caxambu do Sul/SC & $\mathrm{CT}$ & 3369,0 & 21,3 & 36,0 & 49,0 & 70,1 & 17,2 & 10,2 & 5,4 & 0,41 & 9,0 & 7,5 \\
\hline BAF159 & Capinzal/SC & $\mathrm{CT}$ & 2552,0 & 16,4 & 43,0 & 42,0 & 67,2 & 16,6 & 15,3 & 5,1 & 0,51 & 8,3 & 6,8 \\
\hline BAF161 & Lacerdópolis/SC & $\mathrm{CT}$ & 3092,0 & 21,9 & 39,0 & 43,0 & 95,6 & 34,3 & 9,9 & 5,2 & 0,37 & 8,1 & 6,0 \\
\hline BAF164 & Riqueza/SC & $\mathrm{CT}$ & 2499,0 & 19,0 & 41,0 & 51,0 & 81,7 & 30,1 & 8,1 & 4,4 & 0,47 & 11,8 & 8,7 \\
\hline BAF183 & Anchieta/SC & $\mathrm{CT}$ & 3307,0 & 20,0 & 36,0 & 46,0 & 66,6 & 22,4 & 13,3 & 4,7 & 0,46 & 8,8 & 6,7 \\
\hline BAF 184 & Anchieta/SC & $\mathrm{CT}$ & 2789,0 & 43,1 & 31,0 & 37,0 & 78,5 & 18,9 & 8,5 & 3,4 & 0,43 & 8,1 & 7,3 \\
\hline BAF 188 & Palmitos/SC & $\mathrm{CT}$ & 2432,0 & 42,4 & 28,0 & 39,0 & 36,9 & 18,1 & 7,6 & 3,6 & 0,49 & 11,1 & 7,8 \\
\hline BAF189 & Planalto Alegre/SC & $\mathrm{CT}$ & 2891,0 & 18,7 & 36,0 & 50,0 & 77,6 & 19,1 & 14,8 & 4,6 & 0,55 & 10,4 & 8,2 \\
\hline IPR Uirapuru & IAPAR/PR & $\mathrm{CM}$ & 3601,1 & 25,0 & 35,7 & 52,5 & 72,9 & 22,0 & 12,1 & 5,0 & 0,53 & 10,5 & 7,9 \\
\hline BRS Supremo & EMBRAPA/GO & $\mathrm{CM}$ & 3266,8 & 24,5 & 35,4 & 53,3 & 75,4 & 20,6 & 9,2 & 5,5 & 0,52 & 10,4 & 8,0 \\
\hline $\mathrm{QM}^{2 /}$ & - & - & $544378,0^{*}$ & $57,7^{*}$ & $17,0^{*}$ & $22,4 *$ & $197,6^{*}$ & $33,9^{*}$ & $13,7^{*}$ & $0,7 *$ & $0,005^{*}$ & $1,9^{*}$ & $0,9^{*}$ \\
\hline CV (\%) & - & - & 14,3 & 7,7 & 6,8 & 5,8 & 12,8 & 17,6 & 18,9 & 11,1 & 9,40 & 8,2 & 7,3 \\
\hline Média & - & - & 3022,5 & 24,9 & 35,7 & 47,2 & 74,1 & 21,7 & 11,6 & 4,7 & 0,49 & 10,1 & 7,7 \\
\hline
\end{tabular}

${ }^{1 /}$ PROD - Produção de grãos; MCG - massa de cem grãos; DEF - Dias entre a emergência e floração; DFM - Dias entre floração e maturação fisiológica; EP - Estatura de planta; IPL - Inserção do primeiro legume; NLP - Número de legumes por planta; NGL - Número de grãos por legume; DRC - Diâmetro da região do colo; CFT - Comprimento do folíolo principal da terceira folha trifoliolada; LFT - Largura do folíolo principal da terceira folha trifoliolada. ${ }^{2 /}$ Quadrados médios associados aos tratamentos. ${ }^{*}$ Significativo a $5 \%$ de probabilidade pelo teste F. ${ }^{3 /} \mathrm{CT}$ - Cultivar Tradicional (Landrace); CM - Cultivar Melhorada. (IPGRI, 2001). 


\section{Resultados e Discussão}

Na Tabela 1 são apresentadas as médias ajustadas, quadrados médios associados aos tratamentos, coeficiente de variação e média geral dos caracteres avaliados para os 38 acessos e das duas cultivares em estudo pelo procedimento de modelos lineares generalizados (SAS INSTITUTE INC., 1985). Verifica-se diferença significativa entre os tratamentos à significância de $5 \%$ de probabilidade de erro para todos os caracteres avaliados. Os coeficientes de variação demonstram-se baixos conferindo precisão às estimativas (GARCIA, 1989).

A partir do dendrograma, pode ser verificado o agrupamento dos acessos primeiramente em três grupos maiores (Figura 1 e Tabela 2).

O primeiro grupo é compreendido entre os genótipos BAF01 e BAF23, constituído por 26 genótipos, denominado Grupo 1. O segundo grupo compreendido entre os acessos BAF07 e BAF75 (7 genótipos), denominado Grupo 2. O último grupo também formado por sete genótipos entre os acessos BAF08 e BAF47, denominado Grupo 3. Ainda, é possível subdividir os grupos iniciais em subgrupos, uma vez que apresentaram pequenas divergências intragrupos. Assim, dentro do maior grupo (Grupo 1) foram formados 4 subgrupos, sendo o número de genótipos igual a 11, 7, 5 e 3 para os subgrupos 1.1, 1.2, 1.3 e 1.4, respectivamente. O segundo grupo (Grupo 2) foi subdivido em dois subgrupos com 4 e 3 genótipos para os subgrupos 2.1 e 2.2. O Grupo 3 também agregou duas subdivisões com 4 e 3 genótipos para os subgrupos 3.1 e 3.2, respectivamente (Tabela 2). Vale ressaltar que, dentro de cada grupo alguns acessos demonstram-se promissores quanto à característica PROD (Tabela 1). Neste sentido dentro do Grupo 1 destacam se os acessos BAF01 e BAF02, além das testemunhas IPR Uirapuru e BRS Supremo. Já nos Grupos 2 e 3 destacam-se os genótipos BAF07, BAF09 e BAF150 (Grupo 2) e BAF88 e BAF47 (Grupo 3) para o caráter PROD.

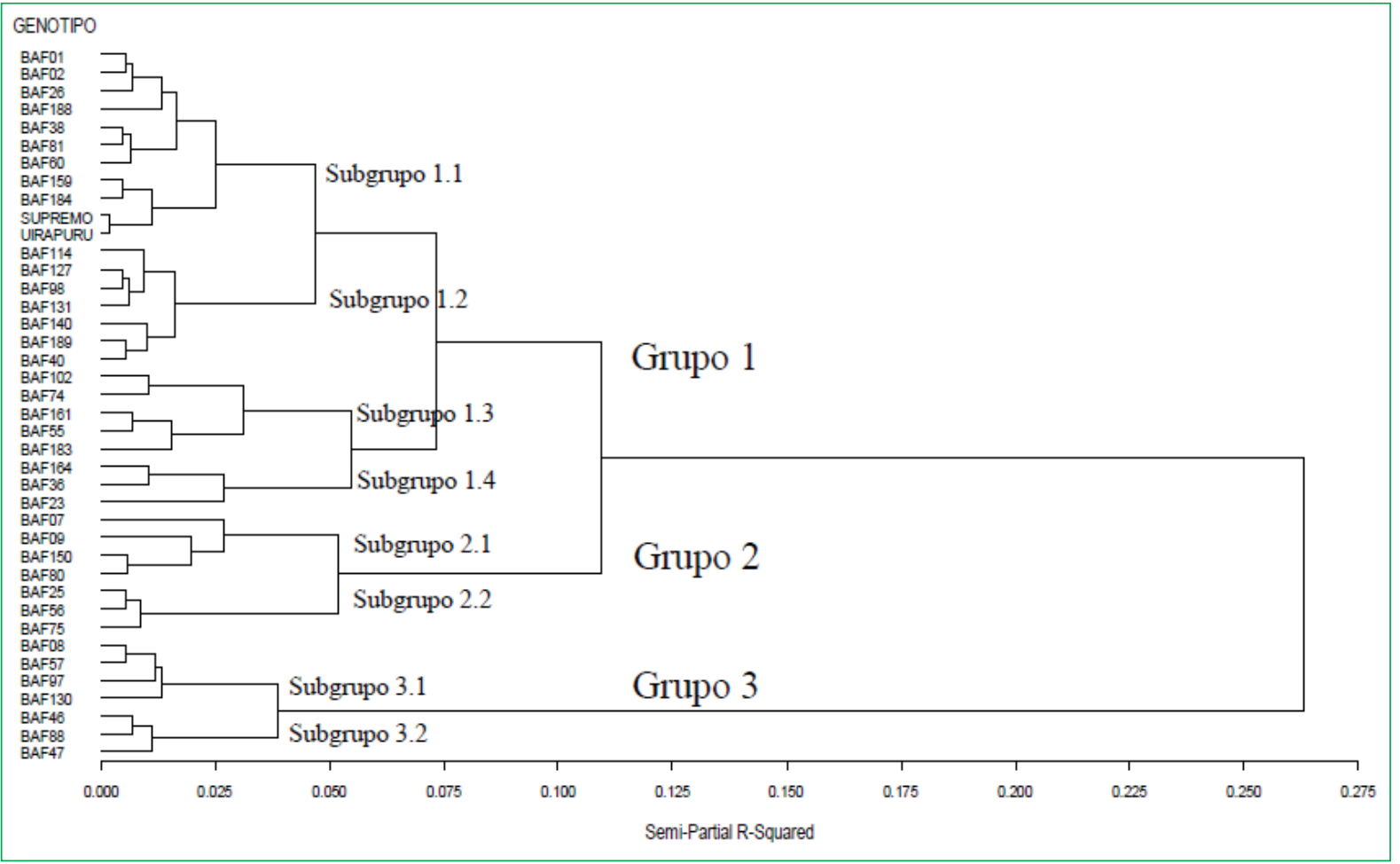

FIGURA 1: Dendrograma de dissimilaridade entre 38 acessos e duas cultivares comerciais de feijão do grupo comercial preto, pertencentes ao Banco de Germoplasma de Feijão do IMEGEM/UDESC, com base na matriz de distância Euclidiana e agrupados pelo método hierárquico de WARD (Lages-SC, 2008/2009). 
TABELA 2: Genótipos agrupados segundo o dendrograma apresentado na Figura 1. (LagesSC, 2008/2009).

\begin{tabular}{|c|c|c|}
\hline \multicolumn{2}{|c|}{ AGRUPAMENTO } & GENÓTIPOS \\
\hline \multirow{4}{*}{$\vec{尺}$} & Subgrupo 1.1 & $\begin{array}{l}\text { BAF01, BAF02, BAF26, BAF188, } \\
\text { BAF38, BAF81, BAF60, BAF159, } \\
\text { BAF184, BRS SUPREMO, IPR UI- } \\
\text { RAPURU }\end{array}$ \\
\hline & Subgrupo 1.2 & $\begin{array}{l}\text { BAF114, BAF127, BAF98, BAF131, } \\
\text { BAF140, BAF189, BAF40 }\end{array}$ \\
\hline & Subgrupo 1.3 & $\begin{array}{l}\text { BAF102, BAF74, BAF161, BAF55, } \\
\text { BAF183 }\end{array}$ \\
\hline & Subgrupo 1.4 & BAF164, BAF36, BAF23 \\
\hline \multirow{2}{*}{$\begin{array}{l}N \\
ٍ \\
ٍ \\
ٍ\end{array}$} & Subgrupo 2.1 & BAF07, BAF09, BAF150, BAF80 \\
\hline & Subgrupo 2.2 & BAF25, BAF56, BAF75 \\
\hline \multirow{2}{*}{ 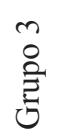 } & Subgrupo 3.1 & BAF08, BAF57, BAF97, BAF130 \\
\hline & Subgrupo 3.2 & BAF46, BAF88, BAF47 \\
\hline
\end{tabular}

Os autores Hair Jr. et al. (2006) ressaltam que a análise de agrupamento utilizando uma distância calculada deve ser utilizada apenas como uma técnica exploratória ou descritiva, já que não possui base estatística sobre a qual se possam realizar inferências.
Os mesmos autores comentam ainda que o agrupamento obtido pela técnica não é único, já que a pertinência a um agrupamento para qualquer número de soluções depende de muitos elementos do procedimento, e muitas soluções diferentes podem ser obtidas pela variação de um ou mais elementos. Sob este ponto de vista, procedeu-se a realização de contrastes multivariados não ortogonais (Tabela 3), tomando como fatores os diferentes grupos formados pelo dendrograma apresentado na Figura 1.

Os contrastes foram efetuados entre os maiores grupos e dentro destes, para a verificação da relevância dos resultados obtidos pela análise de agrupamento. Os resultados demonstram uma alta significância para a maioria dos os contrastes apresentados, inclusive naqueles onde são testadas as menores divergências (intragrupos). No entanto, alguns contrastes não apresentaram significância estatística a $1 \%$ de probabilidade de erro, demonstrando certa ineficiência do método, principalmente em demonstrar diferenças pequenas. Assim, consideram-se relevantes apenas algumas hibridações realizadas com genótipos pertencentes ao mesmo grupo, em subdivisões deste, exceto para os subgrupos 1.1 e $1.2,1.3$ e 1.4 alem dos subgrupos 3.1 e 3.2, os quais não apresentaram diferença estatística a $1 \%$ de probabilidade de erro.

TABELA 3: Contrastes multivariados entre e dentro dos grupos formados com base em distâncias Euclidianas e agrupados pelo método de Ward (Lages-SC, 2008/2009).

\begin{tabular}{lllcc}
\hline \multicolumn{1}{c}{ Contraste } & Teste estatístico & Valor & Valor F & Pr>F \\
\hline Grupo1 vs. Grupo2 & Lambda de Wilks & 0,048 & 26,60 & 0,0001 \\
Grupo1 vs. Grupo3 & Lambda de Wilks & 0,014 & 76,30 & 0,0001 \\
Grupo2 vs. Grupo3 & Lambda de Wilks & 0,012 & 69,82 & 0,0001 \\
Subgrupo 1.1 vs. Subgrupo 1.2 & Lambda de Wilks & 0,249 & 2,74 & 0,0620 \\
Subgrupo 1.1 vs. Subgrupo 1.3 & Lambda de Wilks & 0,087 & 9,54 & 0,0006 \\
Subgrupo 1.1 vs. Subgrupo 1.4 & Lambda de Wilks & 0,154 & 4,99 & 0,0085 \\
Subgrupo 1.2 vs. Subgrupo 1.3 & Lambda de Wilks & 0,062 & 13,75 & 0,0001 \\
Subgrupo 1.2 vs. Subgrupo 1.4 & Lambda de Wilks & 0,106 & 7,68 & 0,0016 \\
Subgrupo 1.3 vs. Subgrupo 1.4 & Lambda de Wilks & 0,212 & 3,38 & 0,0327 \\
Subgrupo 2.1 vs. Subgrupo 2.2 & Lambda de Wilks & 0,142 & 5,47 & 0,0060 \\
Subgrupo 3.1 vs. Subgrupo 3.2 & Lambda de Wilks & 0,166 & 4,56 & 0,0118 \\
\hline
\end{tabular}


Ainda com o intuito de simplificar a estrutura dos dados apresentados na Tabela 1 e agrupar graficamente os acessos mais semelhantes com bases estatísticas mais fortes que aquela apresentada pela análise discriminante, procedeu-se a análise dos dados por meio de variáveis canônicas. É importante salientar que, quando se estuda a diversidade genética pelo método das variáveis canônicas, o propósito é a identificação de genótipos similares em gráficos de dispersão bi ou tri dimensional. Esta técnica possibilita simplificar a interpretação dos resultados, já que tem como fundamento encontrar relações entre dois conjuntos de variáveis, em alta dimensão, em poucos pares de variáveis canônicas (FERREIRA, 1996).

$\mathrm{Na}$ Tabela 4 estão inseridas as variâncias (Autovalores), as variâncias acumuladas das variáveis canônicas calculadas e o coeficiente canônico de ponderação para os caracteres avaliados. Segundo Cruz e Regazzi (1997), para uma interpretação satisfatória da variabilidade manifestada pelos acessos é necessário que as duas primeiras variáveis canônicas permitam a estimativa mínima de $80 \%$ da variação total contida no conjunto de dados analisados. Verificou-se que as duas primeiras variáveis explicam $75,6 \%$ da variação total $(50,3 \%$ pela primeira e $25,3 \%$ pela segunda) (Tabela 4). Quando são consideradas as três primeiras variáveis canônicas, a proporção requerida é alcançada (83\%).
Observa-se também que o caráter MCG foi aquele que mais contribuiu para a dispersão dos genótipos (mais positivos para as primeiras variáveis canônicas), seguido dos caracteres LFT e PROD. Já os caracteres DEF, DFM e IPL foram aqueles que mais contribuíram para o agrupamento dos genótipos (mais negativos para as primeiras variáveis canônicas) (Tabela 4).

Para as três primeiras variáveis canônicas foram calculados os escores para os 38 acessos e para as duas cultivares comerciais em estudo. Estes escores foram plotados em um gráfico de três dimensões, rotacionado de modo a maximizar a visualização das variáveis canônicas que mais explicam a variação contida nos dados (variáveis canônicas 1 e 2).

A avaliação gráfica levando-se em consideração a dispersão gráfica por escores canônicos é bem mais subjetiva que àquela realizada por dendrogramas. No entanto, a partir da Figura 2, onde os escores das duas primeiras variáveis canônicas são posicionados de forma destacada, pode ser visualizada a dispersão diferenciada de alguns genótipos em relação a um grupo maior constituído de diversos genótipos. Verifica-se que o agrupamento obtido pela técnica de variáveis canônicas para os acessos promissores, destacados anteriormente, corrobora e se assemelha aos resultados obtidos na análise de agrupamento com a utilização da distância Euclidiana como parâmetro de divergência, ficando

TABELA 4: Autovalores, variâncias percentuais e variâncias acumuladas em relação às onze variáveis canônicas, para 38 acessos, e duas cultivares de feijão preto oriundas do Banco Ativo de Germoplasma do IMEGEM/UDESC (Lages-SC, 2008/09).

\begin{tabular}{|c|c|c|c|c|c|c|c|c|c|c|c|c|c|}
\hline \multirow{2}{*}{$\begin{array}{l}\text { Variável } \\
\text { canônica }\end{array}$} & \multirow{2}{*}{$\begin{array}{l}\text { Autovalor } \\
\text { (Variância) }\end{array}$} & \multirow{2}{*}{$\begin{array}{c}\text { Variância } \\
\text { acumulada } \\
(\%)\end{array}$} & \multicolumn{11}{|c|}{ Coeficiente Canônico de Ponderação } \\
\hline & & & PROD & MCG & DEF & DFM & EP & IPL & NLP & NGL & DRC & CFT & LFT \\
\hline $1^{\mathrm{a}}$ & 135,8 & 50,3 & 1,73 & 2,69 & $-2,41$ & $-2,05$ & $-0,46$ & $-1,16$ & $-1,23$ & $-0,47$ & 0,75 & $-0,75$ & 1,42 \\
\hline $2^{\mathrm{a}}$ & 68,3 & 75,6 & 1,03 & 0,60 & 1,99 & 2,28 & 1,39 & $-1,64$ & $-2,96$ & 0,14 & $-0,82$ & 1,44 & 1,21 \\
\hline $3^{\mathrm{a}}$ & 20,2 & 83,0 & $-0,29$ & 2,04 & 1,68 & 0,40 & 1,31 & 0,57 & $-0,31$ & $-0,77$ & $-0,65$ & $-0,65$ & 0,45 \\
\hline $4^{a}$ & 19,6 & 90,3 & $-1,63$ & 1,82 & 0,60 & 0,60 & $-1,95$ & 1,59 & 0,63 & 1,76 & $-0,82$ & 3,72 & $-2,76$ \\
\hline $5^{\mathrm{a}}$ & 8,9 & 93,6 & $-0,82$ & $-0,29$ & $-0,35$ & $-0,97$ & $-0,33$ & 0,51 & $-0,44$ & 1,64 & $-0,06$ & $-1,59$ & 1,98 \\
\hline $6^{\mathrm{a}}$ & 6,0 & 95,8 & $-0,02$ & 1,11 & 0,57 & 0,82 & $-0,49$ & 0,54 & $-0,28$ & $-0,66$ & 1,45 & $-0,93$ & 0,84 \\
\hline $7^{\mathrm{a}}$ & 4,2 & 97,4 & 0,78 & $-0,14$ & 0,26 & $-0,62$ & $-0,35$ & 0,40 & $-0,51$ & 0,33 & 0,45 & $-0,07$ & $-0,40$ \\
\hline $8^{\mathrm{a}}$ & 3,2 & 98,6 & 0,42 & 0,03 & $-0,16$ & $-0,71$ & $-0,78$ & 1,05 & 0,99 & 0,53 & $-0,61$ & $-0,02$ & 1,03 \\
\hline $9^{a}$ & 2,0 & 99,3 & 0,14 & 0,69 & $-0,15$ & 0,31 & 0,05 & 0,03 & 0,69 & 0,67 & $-0,18$ & 0,15 & $-0,43$ \\
\hline $10^{\mathrm{a}}$ & 1,1 & 99,7 & $-0,19$ & 0,22 & 0,33 & $-0,55$ & 0,50 & $-0,58$ & 0,04 & 0,19 & 0,40 & 0,58 & $-0,40$ \\
\hline $11^{\mathrm{a}}$ & 0,7 & 100,0 & 0,19 & $-0,64$ & $-0,95$ & $-0,54$ & 0,53 & 0,31 & $-0,14$ & $-0,07$ & 0,30 & 0,21 & 0,00 \\
\hline
\end{tabular}




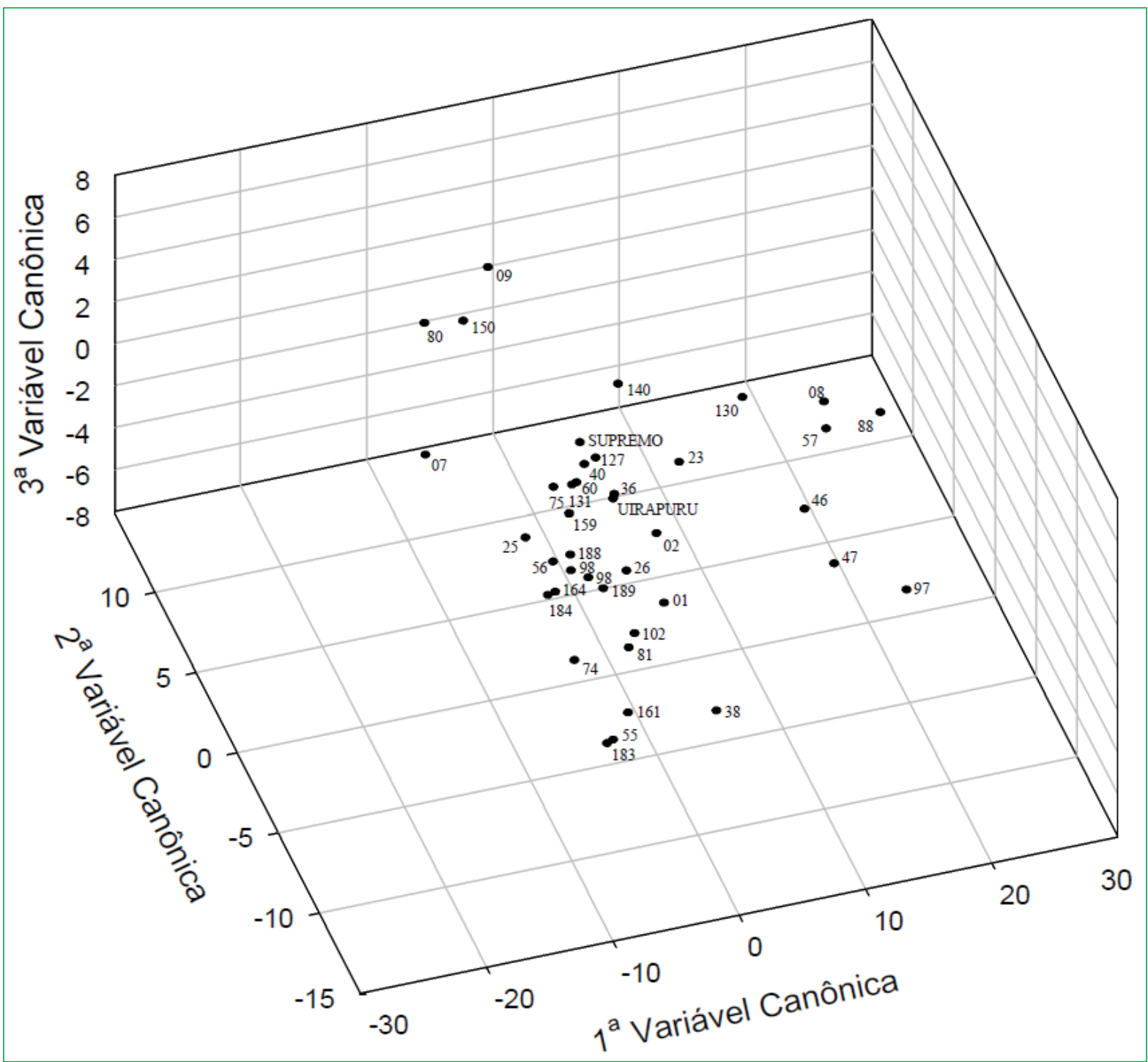

FIGURA 2: Dispersão gráfica dos escores de trinta e oito acessos e duas cultivares comerciais do Banco Ativo de Germoplasma de Feijão da UDESC em relação às três primeiras variáveis canônicas (Lages-SC, 2008/09).

os acessos promissores apresentados anteriormente agrupados de forma similar àquela obtida pelo método proposto por Ward (1963) reforçando a pertinência dos resultados obtidos pelo método anterior, principalmente quando considerados os maiores grupos.

O cruzamento dirigido entre acessos e cultivares pertencentes a grupos contrastantes poderá propiciar a criação de famílias segregantes com elevado potencial produtivo e aumento na probabilidade de recuperar genótipos superiores nas gerações segregantes. Assim, são aconselhados os cruzamentos BAF09/IPR Uirapuru,
BAF09/BRS Supremo, BAF07/IPR Uirapuru, BAF150/ BRS Supremo, entre outros envolvendo acessos e cultivares pertencentes a grupos divergentes.

Verificou-se a existência de variabilidade entre os acessos de feijão preto do Banco Ativo de Germoplasma de Feijão do IMEGEM/UDESC.

A utilização de métodos de análise multivariada adiciona informações relevantes à variação conjunta de variáveis respostas, sendo eficiente na determinação de divergência entre os acessos avaliados. 


\section{Agradecimentos}

Os autores agradecem à UDESC, ao $\mathrm{CNPq}$, à CAPES e à FAPESC, pela concessão de bolsa e apoio financeiro no desenvolvimento deste trabalho.

\section{Referências}

ALLARD, R. W. Principles of plant breeding. 2. ed. New York: John Wiley, 1999. 239 p.

BORÉM, A.; MIRANDA, G. V. Melhoramento de plantas. Viçosa: Editora UFV, 2009. 547 p.

CARVALHO, L. P.; LANZA, M. A.; FALLIERI, J.; SANTOS, J. W. Análise da divergência genética entre acessos de banco ativo de germoplasma de algodão. Pesquisa Agropecuária Brasileira, Brasília, v. 38, p. 1149-1155, 2003.

CARVAlHO FILHO, J.; GOMES, L. A. A.; MALUF, W. R. A. Tolerância ao florescimento precoce e características comerciais de progênies F4 de alface do cruzamento Regina 71 x Salinas 88. Acta Scientiarum Agronomy, Maringá, v. 31, n. 1, p. 37-42, 2009.

CIAT - Centro Internacional de Agricultura Tropical. Sistema estándar para la evaluación de germoplasma de frijól. 2 ed. Cali: CIAT, 1991. 56 p.

COMISSÃO SULBRASILEIRA DE QUÍMICA E FERTILIDADE DO SOLO. Manual de adubação e calagem para os estados do Rio Grande do Sul e Santa Catarina. Porto Alegre: SBCS/Núcleo Regional Sul, 2004. 400 p.

CRUZ, C. D. Aplicação de algumas técnicas multivariadas no melhoramento de plantas. 1990. $188 \mathrm{f}$. Tese (Doutorado em Genética e Melhoramento de Plantas) - Escola Superior de Agricultura Luiz de Queiroz, Piracicaba, 1990.

CRUZ, C. D.; CARVALHO, S. P.; VENKOVSKY, R. Estudos sobre divergência genética I. Fatores que afetam a predição do comportamento de híbridos. Revista Ceres, Viçosa, v. 41, p. 178182, 1994a.

CRUZ, C. D.; CARVALHO, S. P.; VENKOVSKY, R. Estudos sobre divergência genética II. Eficiência da predição do comportamento de híbridos com base na divergência genética de progenitores. Revista Ceres, Viçosa, v. 41, p. 183-190, 1994 b.

CRUZ, C. D.; REGAZZI, A. J. Modelos biométricos aplicados ao melhoramento genético. Viçosa: Editora UFV, 1997. 390 p.

DIAS, L. A. S.; KAGEYAMA, P. Y. Multivariate genetic divergence and hybrid performance of cacao (Theobroma cacao L.). Brazilian Journal of Genetics, Ribeirão Preto, v. 20, n. 1, p. 63-70, 1997.

EMBRAPA. Sistema brasileiro de classificação de solos. Brasília: Centro Nacional de Pesquisa de Solos, 1999. 412 p.

FALCONER, D. S.; MACKAY, J. F. C. Introduction to quantitative genetics. 4. ed. London: Longman Malayse, 1996. 464 p.

FEDERER, W. T. Augmented (hoonuiaku) designs. Hawaiian Planters' Record, Kunia, v. 55, p. 191-208, 1956.

FERREIRA, D. F. Análise multivariada. Lavras: Universidade Federal de Lavras, 1996. 400 p.

FIORINI, C. V. A.; GOMES, L. A. A.; MALUF, W. R.; FIORINI, I. V. A.; DUARTE, R. P. F.; LICURSI, V. Avaliação de populações F2 de alface quanto à resistência aos nematóides das galhas e tolerância ao florescimento precoce. Horticultura Brasileira, Campinas, v. 23, n. 2, p. 299-302, 2005.

GARCIA, C. H. Tabelas para classificação do coeficiente de variação. Piracicaba: Circular Técnica no 171 - IPEF, 1989. 11 p.

HAIR JR., J. F.; ANDERSON, R. E.; TATHAM, R. L.; BLACK, W. C. Análise multivariada de dados. 5 ed. Porto Alegre: Bookman, 2006. 600 p.

IPGRI - International Plant Genetic Resources Institute. Descritores para Phaseolus vulgaris L. Roma: IPGRI, 2001. 45 p.

KHATTREE, R.; NAIK, D. N. Applied multivariate statistics with SAS ${ }^{\circledR}$ Software. 2. ed. Cary: SAS Institute Inc., 1999. 338 p. KUREK, A. J.; CARVALHO, F. I. F.; OLIVEIRA, A. C.; MARCHIORO, V. S.; CRUZ, P. J. Fatores genéticos relacionados com a expressão do caráter percentual de cariopse em aveia branca. Ciência Rural, Santa Maria, v. 32, n. 5, p. 751-756, 2002.

MATEI, G. Implicações da capacidade de combinação e da distância genética na seleção de genitores de trigo (Triticum aestivum L.). 2010. 81 f. Dissertação (Mestrado em Agronomia) - Universidade Tecnológica Federal do Paraná, Pato Branco. 2010. MENDONÇA, H. A.; SANTOS, J. B.; RAMALHO, M. A. P. Selection of common bean segregating populations using genetic and phenotypic parameters and RAPD markers. Crop Breeding and Applied Biotechnology, Viçosa, v. 2, p. 219-226, 2002.

MINGOTI, S. A. Análise de dados através de métodos de estatística multivariada: uma abordagem aplicada. Belo Horizonte: Editora UFMG, 2005. 297 p.

RONALD, P. S.; BROWN, P. D.; PENNER, G. A. Heritability of hull percentage in oat. Crop Science, Madison, v. 39, p. 5257, 1999.

SARTORIO, S. D. Aplicações de técnicas de análise multivariada em experimentos agropecuários usando o software R. 2008. 130 f. Dissertação (Mestrado em Agronomia) - Escola Superior de Agricultura 'Luiz de Queiroz', Piracicaba, 2008. 130 p.

SAS INSTITUTE INC. SAS User's Guide: statistics. 5 ed. Cary: SAS Institute Inc., 1985. 956 p.

STATISTICAL ANALYSIS SYSTEM. SAS: Statistical Analysis System - Getting Started with the SAS ${ }^{\circledR}$ Learning Edition. Cary: SAS Institute Inc., 2002. 86 p.

SILVA, R. R.; GOMES, L. A. A.; MONTEIRO, A. B. I.; MALUF, W. R.; CARVALHO FILHO, J. L. S.; MASSAROTO, J. A. Linhagens de alface-crespa para o verão resistentes ao Meloidogyne javanica e ao vírus mosaico-da-alface. Pesquisa Agropecuária Brasileira, Brasília, v. 43, n. 10, p. 1347-1356, 2008.

SILVA, H. T. Descritores mínimos indicados para caracterizar cultivares/variedades de feijão comum (Phaseolus vulgaris $\mathbf{L}$.$) .$ Santo Antônio de Goiás: Embrapa Arroz e Feijão, 2005. 32 p.

WARD, L. H. Hierarchical grouping to optimize an objective function. Journal of the American Statistical Association, Alexandria, v. 58, p. 236-244, 1963.

WESP, C. L.; MARTINELI, J. A.; CHAVES M. S.; GRAICHEN, F. A. S.; FEDERIZZI, L. C. Herança da resistência quantitativa à ferrugem da folha em linhagens recombinantes de aveia branca. Tropical Plant Pathology, Brasília, v. 33, n. 2, p. 138-147, 2008. 